
CRUSTACEA IIBRARX

SMITHSONIAN INST.

RETURN IO W-119 



\section{LA FEUILLE}

\section{DES JEUNES NATURALISTES}

REVUE MENSUELLE D'HISTOIRE NATURELLE Fondée à Mulhouse en 1870

\section{PRIX DE TABONNEMENT}

Payable à M. Adrien DOLLFUS, 35, rue Pierre-Charron, Paris

France et Étranger .............................. fr. 4 par an Avec cotisation supplémentaire pour jouir de la Bibliothèque (France).. fr. 8 par an Le Numéro, 40 centimes.

LES ABONNENENTS CONPTENT a PARTIR DU for NOVENBRE DE CHAQUE AVNÉE
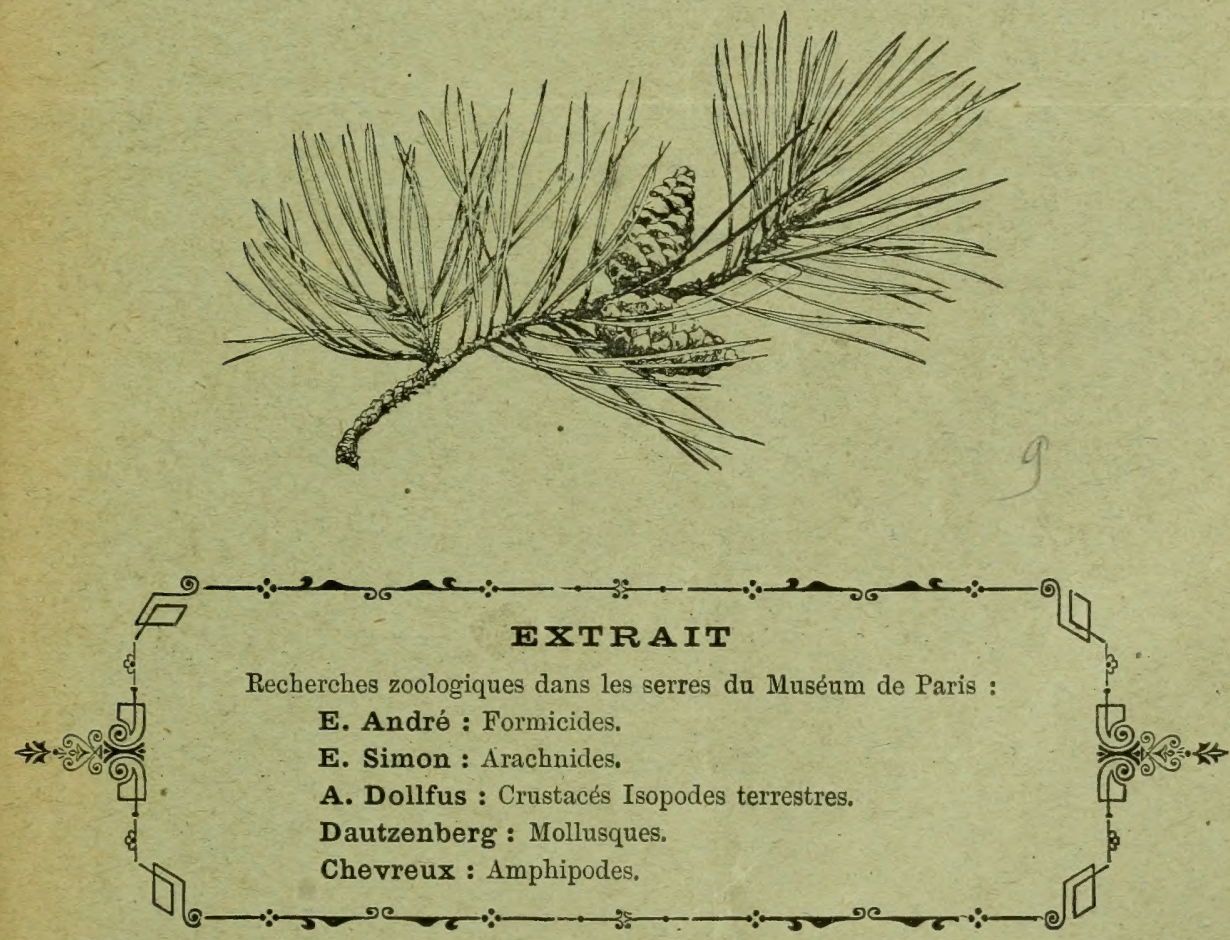

TYP. OBERTHUR, A BENNES - MAISON A PARIS rue Salomon-de-Caus, 4 (square des Arts-et-Métiers) 



\section{RECHERCHES ZOOLOGIQUES}

\section{DANS LES SERRES DU MUSÉUM DE PARIS ${ }^{(1)}$}

Introduction. - La faune exotique acclimatée dans les serres chaudes a depuis longtemps attiré l'attention des zoologistes. Un assez grand nombre d'espèces exotiques vivant ainsi dans des conditions plus ou moins anormales ont dejà été signalées en divers endroits; ces espèces se retrouvent souvent dans des serres différentes; elles s'y propagent et leur présence ne peut être considérée comme purement accidentelle; ce sont en général des animaux qui ont dans les pays chauds une dispersion très vaste, et par conséquent une assez grande faculté d'acclimatation; à cette faune importée se joignent des espèces indigènes venant du dehors, et d'autres, dont le milieu normal se trouve précisément en ces lieux clos, humides, chauds et riches en humus. Il y a donc en somme deux faunes intéressantes à signaler dans les serres : une faune propre et une faune récemment acclimatée. Ne peuvent être considérées comme leur appartenant, ni les espèces indigènes venant du dehors, ni certaines formes tropicales introduites accidentellement avec des plantes exotiques et qui, n'étant pas susceptibles d'acclimatation, ne se propagent pas et sont destinées à disparaître rapidement.

Nous avons pensé qu'il serait intéressant d'entreprendre dans les serres du Jardin des Plantes, dans lesquelles s'accumulent depuis si longtemps tant de plantes de provenances diverses, une série de nouvelles recherches méthodiques pour en établir la faunule. Grâce à l'obligeance de M. le professeur Cornu qui a bien voulu nous autoriser à faire ces recherches, et de M. le professeur Bouvier qui nous a prêté sa collaboration active, nous avons pu, en novembre et décembre derniers, faire ainsi d'intéressantes découvertes dont nous donnons ci-dessous le résultat.

MM. Bouvier, Bedel, Brolemann, E. Simon et Ad. Dollfus formaient la petite phalange d'exploration, secondée par le personnel des serres. Nous avons eu recours également à MM. Ernest André (de Gray), Chevreux (de Bône) et Dautzenberg pour la détermination des groupes qui n'étaient pas de notre compétence.

Un fait assez curieux que nous avons eu l'occasion de constater, c'est l'absence, jusqu'à présent complète, d'insectes tropicaux acclimatés, en dehors des Formicides et des Coccides. Par contre, les Myriapodes, les Crustacés et les Arachnides nous ont réservé des surprises auxquelles nous ne nous attendions pas.

\section{Ad. Dollfus.}

\section{I. - Formicides}

1. Lasius flavus Fab. Espèce très commune en France et abondamment répandue dans la majeure partie de la région paléarctique ainsi que dans l'Amérique du Nord.

2. Prenolepis longicornis Latr. Espèce importée et qui s'est acclimatée depuis longtemps dans quelques serres chaudes des grandes villes d'Europe.

(1) Une note préliminaire sur nos recherches a paru dans le Bulletin du Muséum (1896, $\mathrm{n}^{\mathrm{o}}$ 1). M. le prof. Bouvier a fait une communication à ce sujet, à la réunion des Naturalistes du Muséum, le 28 janvier 1896. 
Elle vit à l'état autochtone en Egypte, en Syrie et dans les régions tropicales des deux mondes.

3. Ponera coarctata Latr. Se trouve en petites fourmilières isolées et assez cachées, en France, dans presque toute la région paléarctique et dans l'Amérique du Nord.

4. Triglyphotrix obesa André, race striatidens Em. Cette fourmi, décrite originairement sur des exemplaires de l'Inde et de la Birmanie, a été retrouvée depuis à Sierra Leone et même en Tunisie, mais sa présence n'avait pas encore été signalée dans les serres d'Europe.

5. Pheidole megacephala Fab. Ne se trouve pas en France sous sa forme typique, mais c'est une espèce extrêmement répandue dans les parties les plus chaudes de la région paléarctique ainsi que dans tontes les contrées tropicales ou subtropicales du monde entier. Comme tous les Pheidole, elle possède deux castes de neutres bien distinctes : les ouvrières et les soldats.

Pour permettre la facile reconnaissance de cette faunule myrmécologique des serres du Muséum, je résume, sous forme de tableau, les principaux caractères des cinq espèces précédentes qui n'étaient représentées que par des neutres.

A. Pétiole de l'abdomen composé d'un seul article surmonté d'un appendice en forme d'écaille verticale ou oblique.

a. Abdomen proprement dit (abstraction faite du pétiole) non étranglé entre son premier et son second segment.

- Forme courte, thorax à peu près de la longueur de l'abdomen; écaille du pétiole mince et assez élevée, peu inclinée, yeux relativement très petits, pattes et antennes courtes, scape dépassant seulement un peu l'oceiput quand il est ramené en arrière. Tout le corps mat, d'un jaune rougeâtre, non hérissé de longs poils. Long. 3-3 1/2 mill.

Lasius flavus Fab.

- Forme étroite et très allongée; thorax grêle et beaucoup plus long que l'abdomen, écaille du pétiole plus épaisse, basse et fortement inclinée en avant; yeux relativement grands, pattes et antennes très longues; scape dépassant l'occiput de plus de moitié de sa longueur. Tout le corps luisant, d'un brun noir, hérissé de longs poils épars. Long. 21/2-3 mill.

Prenolepis longicornis Latr.

b. Abdomen proprement dit sensiblement étranglé entre son premier et son second segment. Corps cylindrique, allongé, assez luisant, écaille du pétiole épaisse, verticale; yeux extrêmement petits et peu distincts. Antennes et pattes courtes et robustes, couleur variant du brun noir au jaune rougeâtre. Long. 2 1/2-3 mill. Ponera coarctata Latr.

B. Pétiole de l'abdomen composé de deux articles plus ou moins nodiformes. a. Second article du pétiole à peine plus large que le premier. Tête, thorax et pétiole mats, fortement ponctués-réticulés ou ridés-réticulés, métanotum armé en arrière de deux longues épines aiguës, dirigées horizontalement, et de deux autres plis courtes, en dessous des premières, de chaque côté de l'articulation du pétiole. Couleur d'un brun rougeâtre avec l'abdomen plus foncé, les pattes et les antennes plus pâles. Long. 2-2 1/2 mill.

Triglyphothrix obesus André, race striatidens Em.

b. Second article du pétiole beaucoup plus largé que le premier, tout le corps luisant, presque lisse, métanotum armé seulement de deux courtes épines aiguës et verticales. Couleur d'un brun marron foncé avec les antennes et les pattes, en majeure partie, d'un testacé pâle. Le soldat a la tête énorme, échancrée en arrière, au moins deux fois aussi large que le thorax, striée sur sa moitié antérieure, avec le scape 
des antennes court, étant loin d'atteindre l'occiput; sa taille est de 4-4 1/2 millim. L'ouvrière a la tête normale, non échancrée en arrière, entièrement lisse et luisante, avec le scape des antennes dépassant très notablement l'occiput, quand il est ramené en arrière; sa taille est de 2-2 1/2 mill.

Pheidole megacephala Fab.

\section{Nole supplémentaire.}

Une seconde visite aux serres du Muséum, effectuée le 25 décembre dernier, a procuré le mâle du Prenolepis longicornis trouvé en compagnie de ses ouvrières. Voici son signalement abrégé qui permettra de le reconnaître facilement.

Antennes et pattes longues et grêles, scape beaucoup plus long que la moitié du funicule. Corps luisant, d'un brun jaunâtre sale; abdomen plus foncé, organes génitaux grands et saillants. Ailes hyalines avec une seule cellule cubitale, sans cellule discoïdale. Long. 2 1/2 mill.

Gray.

Ernest ANDRE.

\section{II. - Araghnides}

1. Schizonotus (Nyctalops) tenuicauda Cambr. (Ann: Mag. Nat. hist., sec. 4, vol. X, 1872, p. 4, pl. XXII, f. 2).

Se trouve assez communément dans les serres du Muséum, sous les pots reposant sur le gravier; il court avec une étonnante agilité et est très difcile à saisir.

Cette espèce a été décrite de Ceylan, par Cambridge; nous l'avons retrouvée depuis, à Singapore, et elle existe probablement en Birmanie, car le Tripeltis Grassii Thorell (Ar. Artrog. Birm., 1889, p. 38) pourrait en être synonyme.

Le genre Schizonolus Thorell (décrit antérieurement par Cambridge, sous le nom de Nyctalops, faisant double emploi) est le type d'un sous-ordre de l'ordre des Pedipalpes, se rapprochant surtout des Thelyphones dont il représente la forme amoindrie; il est principalement caractérisé par son céphalothorax, divisé en deux segments, dont le second plus court que le premier, est souvent divisé lui-même par une strie membraneuse longitudinale et par ses pattes-mâchoires, terminées en crochet simple, nullement en pince didactyle.

On en connaît trois espèces propres à l'Asie tropicale et à la Malaisie; nous en avons trouvé deux autres (inédites) au Venezuela, et le professeur Grassi en a signalé une de Sicile, sous le nom de Konenia mirabilis (Naturalista Siciliano, IV, 1885, p. 127).

\section{Ischnothyreus lymphaseus E. Sim.}

Cette petite espèce de la famille des Oonopides, que nous avons découverte a Ceylan, dans les détritus humides des forêts, se trouve communément sous les pots de semis des serres du Muséum; son agilité est très grande, ce qui la rend difficile à saisir, au moins intacte.

Le genre Ischnothyreus se distingue des autres types du groupe des Oonopides cuirassés par ses yeux antérieurs connés et ses scuta abdominaux très raccourcis, surtout le ventral qui ne dépasse pas le pli épigastrique, et par ses pattes antérieures, armées d'épines sériées fines et couchées. L'I. lymphaseus appartient au premier groupe de ce genre qui se distingue des suivants par des caractères sexuels, le céphalothorax du mâle étant beaucoup plus convexe que celui de la femelle et sa patte-mâchoire étant très petite et incurvée; ce groupe renferme deux autres espèces : l'I. bipar- 
titus E. Sim., également de Ceylan et l'I. aculeatus E. Sim. des Philippines.

Une espèce du second groupe, qui se rencontrera probablement aussi dans les serres chaudes, l'I. peltifer E. Sim., est remarquable par sa grande dissémination, car nous la possédons de l'Atrique occidentale, de Ceylan, des Philippines et des Antilles.

\section{Triceris stenaspis E. Simon.}

Se trouve sur le sol, en même temps que le Schizonotus. Comme l'Ischnothyreus, cette petite espèce appartient au groupe des Oonopides cuirassés, mais elle est d'origine américaine : découverte au Venezuela, elle a été retrouvée depuis aux Antilles. Les scuta abdominaux du Triceris sont très courts, comme ceux des Ischnothyreus, mais ses yeux ressemblent davantage à ceux des Gamasomorpha, les antérieurs étant largement disjoints; ses pattes antérieures, également armées de fines épines sériées, sont remarquables par la grande longueur de leur article patellaire.

\section{Theridion (Coleosuma) blandum Cambr.}

Ce petit Theridion, décrit de Ceylan, retrouvé depuis à Singapore et aux Philippines et indiqué de la Floride, par Keyserling, est assez commun dans les serres du Muséum; ses allures et ses mœurs sont tout à fait différentes de celles des espèces précédentes; il vit, en effet, sur les plantes au milieu d'un petit réseau très simple. Au moment de la ponte, la femelle porte son cocon qui est gros, blanchâtre et floconneux, suspendu aux filières, comme le font les autres espèces du groupe du Theridion bimaculatum L.

Dans cette espèce, les sexes sont très dissemblables, la femelle est normale et son abdomen est presque globuleux, tandis que l'abdomen du mâle, étroit, allongé et un peu renflé en arrière, est remarquable par le grand développement de sa région épigastrique, s'avançant en forme de cou cylindrique a la rencontre du céphalothorax et ressemblant un peu au pédicule d'une fourmi. Cambridge, qui n'a connu que le mâle, en avait fait le type d'un genre Coleosoma, qui n'a pas été maintenu, le caractère sur lequel il repose étant uniquement sexuel.

Paris.

Eugène Srmon.

\section{III. - Crustacés Isopodes terrestres}

Le nombre des espèces de crustacés Isopodes terrestres, recueillies par nous dans les serres du Jardin des Plantes, est de dix, dont voici la liste :

Armadillidium nasatum $\mathrm{BL}$.;

Porcellio pictus Br.;

- dilatatus Br.;

- scaber Latr.;

- lovvis Latr.;

Metoponorthus pruinosus Br. (Porcellio).

Bathytropa thermophila (nova species);

Oniscus murarius Cuv.;

Haplophthalmus Danicus BL.;

Trichoniscus roseus Koch.

Nous ne croyons pas qu'aucune de ces espèces puisse être considérée comme introduite de pays exotiques : Metoponorthus pruinosus, espèce ubiquiste, appartient aussi bien à notre faune indigène qu'aux pays tropicaux où elle pullule en bien des localités; Trichoniscus roseus est particulièrement abondant dans les serres du Muséum, et l'intensité de sa coloration rouge est 
extrême; c'est une espèce commune dans les endroits humides et obscurs.

Haplophthalmus Danicus BL. (= II. Hengii M. Weber) est un petit Isopode d'un blane jaunâtre ou grisâtre qui paraît abondant dans le terreau des serres et des jardins; on l'a signalé en Danemark, en Allemagne, en Hollande, et nous l'avions déjà rencontrée abondamment dans le terreau du jardin zoologique de La Haye (Hollande) et dans une serre à Châtillonsur-Bagneux (Seine).
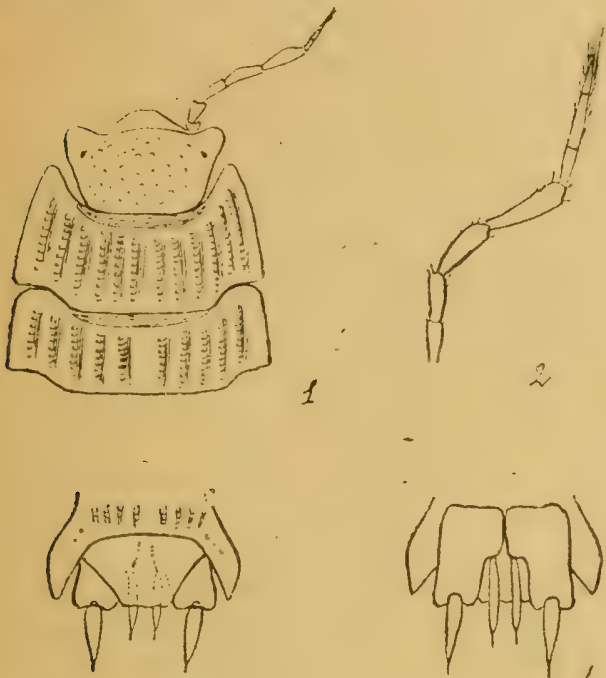

3

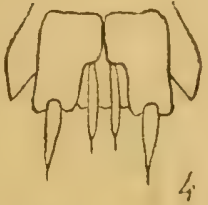

FIG. 1. - Haplophthalmus Danicus BL.

1. Partie antérieure : Céphalon et deux premiers segments du péreion (vus en dessus).

2. Antenne.

3. Partie postérieure : Јc segment clı pléon, pléotelson et uropodes (vus en dessus).

4. Id. (vus en dessous).

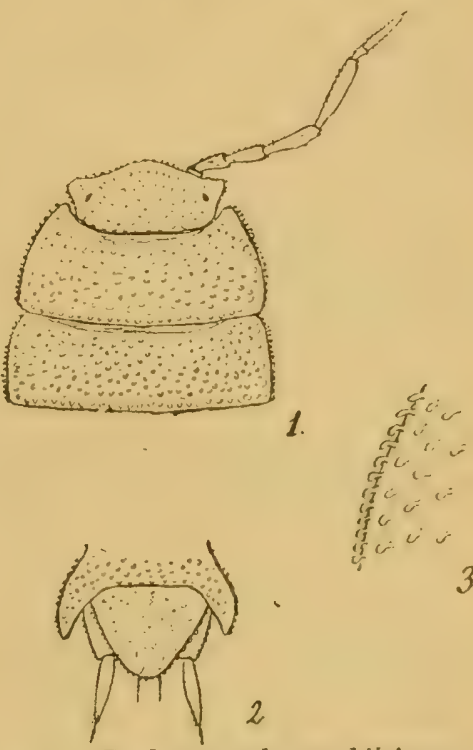

FIG. 2.-Bathytropa thermophilanova sp.

1. Partie antérieure : Céphalon et deux premiers segments du péreion (vus en (lessus).

2. Partie postérieure : 5 segment Łlu pléon, pléotelson et uropodes (v, en dessous).

3. Poils écailleux du péreion.

Bathytropa thermophila est une espèce nouvelle très intéressante dont voici une courte diagnose : corps ovale allongé couvert de poils écailleux; céphalon à lobe méaiian largement arrondi, lobes latéraux petits; yeux très petits, antennes à fouet biarticulé, le premier article trois fois plus court que le second; premier segment du péreion à bord postérieur non sinueux; pléotelson aussi long que large, à sommet arrondi ; base des uropodes n'atteignant pas l'extrémité du pléotelson; exopodites dépassant sensiblement celui-ci; endopodites atteignant le sommet du pléotelson. Couleur : blanchâtre. Dimensions : 2 millimètres $1 / 4$ sur 1 millimètre.

Cette espèce appartient à un genre considéré comme exclusivement méditerranéen et qui comprenait, jusqu'à présent, quatre espèces décrites; l'habitat précis de la plupart de celles-ci (Bathytropa .Ineinertii BL., d'Algérie; $B$. costata BL., d'Algérie et de Porquerolles; $B$. hispana Dollfus, de Valence) n'a pas été signalé, mais une autre espèce qui nous a été envoyée par M. Lubert, de Marseille (B. gramulata Dollfus) a été recueillie dans un jardin, au pied d'un mur et is une certaine profondeur dens la terre. - Il est très probable que tout ce genre se compose d'espèces plus ou moins hypogées, c'est bien le cas de celle que nous décrivons aujourd'hui et que nous avons recueillie en grattant le gravier et la terre qui forment le sol de l'une des serres chaudes, ainsi que dans le terreau des pots de fleurs. 


\section{$-6-$ \\ IV. - Mollusques}

La récolte faite dans les serres du Jardin des Plantes comprend sept espèces de Mollusques, dont quatre indigènes:

Hyalinia lucida Müller, nitida Müller,

Patula rotundata Müller, Physa acuta Draparnaud,

et trois exotiques. Celles-ci appartiennent toutes au même genre Stenogyra:

1. Stenogyra (Opeas) Goodalli Miller.

Ce Mollusque, originaire des Antilles, est particulièrement abondant dans les serres du Muséum. Son acclimatation a déjà été signalée en Angleterre, notamment à Bristol. Le Stenogyra muscecola Morelet, du Gabon, est extrêmement voisin du Goodalli, si même, il ne lui est identique.

\section{Stenogyra (Opeas) octonoïdes C. B. Adams.}

Beaucoup plus rare que la précédente dans les serres du Muséum, cette espèce est originaire des Antilles et de la Guyane.

\section{Stenogyra (Spiraxis) venusta Morelet.}

C'est avec quelque doute que nous désignons ainsi la troisième espèce recueillie, car il ne nous a pas été possible de trouver cette dénomination dans les diverses publications de Norelet. Tout ce que nous pouvons affirmer, c'est que les deux exemplaires que nous avons sous les yeux, concordent sous tous les rapports avec ceux qui ont été recueillis en 1860 par Eudel à Saint-Pierre de la Réunion, et que Deshayes a déterminés ainsi a cette époque. Il s'agit probablement là d'un nom resté à l'état manuscrit.
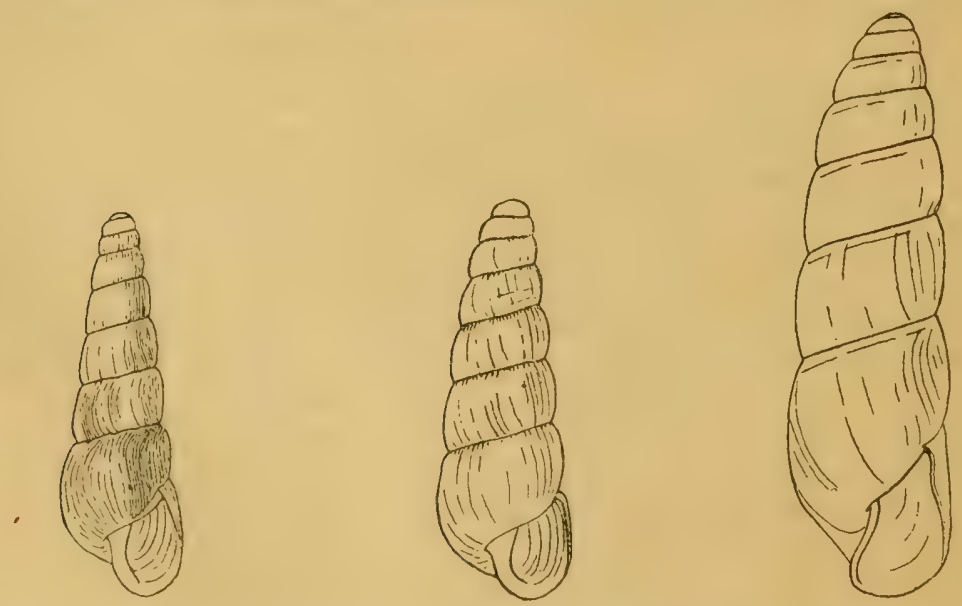

Cette espèce ressemble assez, au premier aspect, au Stenogyra (Subulina) octona Chemnitz; mais il suffit d'examiner de près la conformation de sa columelle pour s'assurer qu'elle appartient à une section différente.

En effet, tandis que la columelle est tronquée à la base chez le St. octona, elle est tordue et non tronquée chez le Stenogyra (Spiraxis) venusta.

Ph. Dautzenberg. 


\section{V. - Sur un Amphipode terrestre exotique,}

Talitrus Alluaudi nov. sp.,

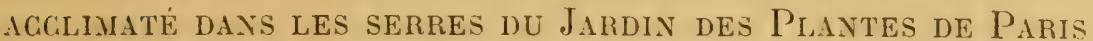

Vers la fin de l'année dernière, M. Adrien Dollfus a eu l'obligeance de m'envoyer, à deux reprises différentes, des exemplaires d'un Amphipode recueilli dans le terreau et le gravier humide des serres chaudes du Jardin des Plantes de Paris. Le premier envoi ne contenait que de très jeunes spécimens, appartenant sans conteste à la famille des Oichesticlie, mais qu'il n'était guère possible de classer, même comme genre. Quelques jours plus tard, je recevais une trentaine d'exemplaires adultes, parmi lesquels un certain nombre de femelles ovifères, et je pouvais identifier la forme du Jardin des Plantes avec un Talitre nouveau, qui fait partie d'une petite collection d'Amphipodes, rapportée des îles Séchelles, en 1892, par M. Charles Alluaud. Je suis heureux de dédier cette nouvelle espèce à M. Alluaud, qui m'a confié l'étude des Amphipodes recueillis pendant son voyage.

Talitrus Alluaudi est la plus petite espèce connue du genre (1); les plus grandes femelles ovifères ne dépassent pas 6 millim. de longueur; les mâles atteignent, au plus, 7 millim. Le dimorphisme sexuel est, du reste, très peu accentué, et consiste seulement dans les proportions du pédoncule des antennes inféricures, plus robuste et un peu plus long chez le inâle que chez la femelle. En dehors de leur petite taille, ces Talitres sont bien caractérisés par la grande longueur de leurs antennes supérieures, par la forme allongée de l'article basal des pattes des cinquième et sixième paires, et surtout par l'état rudimentaire de leur dernière paire d'uropodes.

Voici une courte description de l'espèce :

Corps peu comprimé. Epimères antérieurs notablement moins lauts que les segments correspondants du thorax, ceux de la cinquième paire, très grands, atteignant la hauteur des précédents. Yeux assez grands, arrondis. Antennes supérieures (fig. 1), très longues, dépassant le milieu du dernier article du pédoncule des inférieures; flagellum aussi grand que le pédoneule,

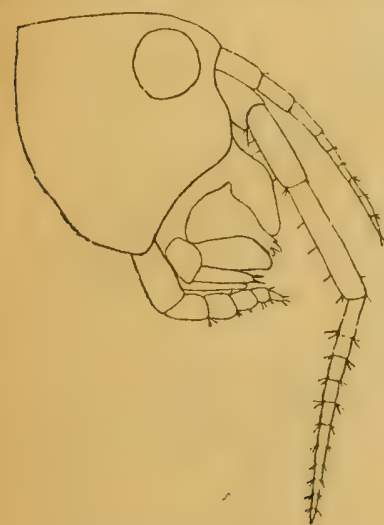

FIG. 1.

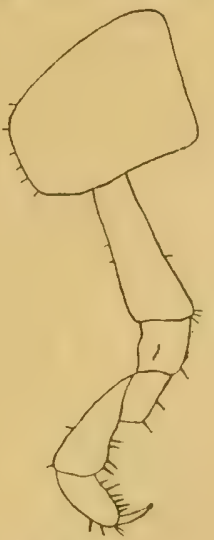

FIG. 2.

T. Alluardi $\sigma^{\top} .-$ Tête et antennes, $\times 27$.

T. Alluaudi o. - Gnathopode antérieur $\times 36$.

(1) L'Orchestio fissispinosa Kossmann (Zoologische Ergebnisse eincr Reise in die Kustengebiete des Rothen Meeres, Leipzig, 1880), décrite d'après un unique spécimen de 5 millim. de longueur, doit être un très jeune exemplaire de Talitrus, de Talorchestia oul d'Orchestoidla. La tigure (Pl. XIII, fig. 1) représentant l'antenne supérieure, qui comprend en tout cinq articles, montre bien qu'il ne s'agit pas d'une forme adulte. 
et comprenant six articles assez allongés. Pédoncule des antennes inférieures un peu plus long et plus gros chez le mâle que chez la femelle; troisième article plus allongé que d'ordinaire; quatrième article beaucoup plus court que le cinquième; flagellum notablement moins long que le pédoncule, et comprenant de neuf à onze articles (Chez les femelles, les antennes inférieures sont à peine plus longues que l'ensemble de la tête et des deux premiers segments du thorax).

Masse buccale très proéminente. Pièces buccales ne différant pas sensiblement de celles du type ( $T$. locusta). Gnathopodes antérieurs (fig. 2) très robustes; carpe renflé à sa partie inférieure; propode gros et court, garni de longues épines au bord interne.

Carpe des gnathopodes postérieurs (fig. 3) très court, à peine plus long que le propode, qui présente une extrémité large et arrondie; dactyle très éloigné de l'extrémité du propode.

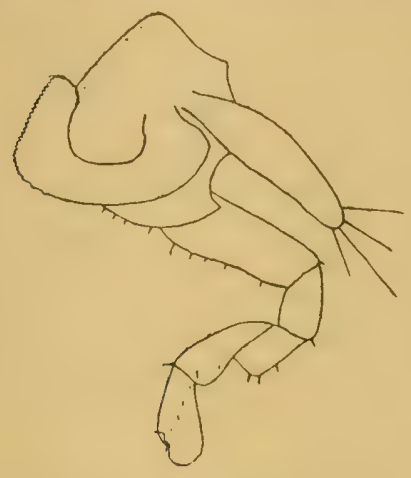

FIG. 3 .

T. Alluuvdi Q. - Gnathopode postérieur $\times 36$.

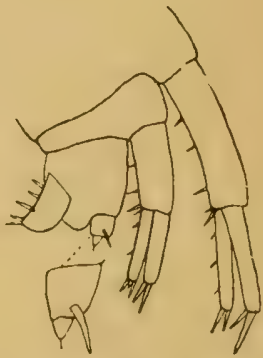

FIG, 4.

T. Alluandi $9 .-$ Uropodes et telson $\times 27$.

Pattes des deux dernières paires courtes et robustes. Article basal étroitement ovale chez les pattes des cinquième et sixième paires, presque circulaire et denticulé au bord postérieur chez les pattes de la septième paire. Uropodes des deux premières paires normaux. Uropodes de la troisième paire (fig. 4) rudimentaires, mesurant à peine la moitié de la longueur du telson; pédoncule armé, à sa partie inférieure, d'une longue épine recourbée; branche extrêmement petite, conique, atteignant à peu près la moitié de la longueur du pédoncule, et portant une soie courte a son extrémité. Telson très volumineux, quadrangulaire, aussi long que large, ne présentant aucune trace d'échancrure, armé de dix grandes épines marginales : quatre de chaque côté, deux au bord postérieur.

Longueur : ơ 7 millim. - o 5 à b̉ millim.

Ces 'Talitres sautent, paraît-il, avec une grande agilité, et sont assez. difficiles à saisir. Aux Séchelles, M. Alluaud les a recueillis dans plusieurs localités différentes de l'île de Mahé, tantôt au bord des marigots, dans des trones pourris de cocotiers, tantôt dans l'humus des forêts.

Bône.

E. Chevreux.

Extrait de la Feuille des Jeunes Naturalistes, 35, rue Pierre-Charron, Paris Abonnement avec Bibliothèque (France), 8 fr. - Sans Bibliothèque (France et Étranger), 4 fr. 



\section{BULLETIN D'ÉCHANGES DE LA FEUILLE DES JEUNES NATURALISTES}

La Société des Jeunes Naturalistes de Tournus (Saône-et-Loire) demande à échanger des plantes de la région contre d'autres plantes de France et plus spécialement de la région lyonnaise. Adresser les offres au président de la Société.

M. J. Courjault, instituteur, Saint-Genis-de-Saintonge (Charente-Inférieure), offre plantes (400 esp.), roches et minciraux (250 esp.), fossiles (350 esp), contre fossiles primaires, triasiques, tertiaires, sauf éocène, exclusivement de France, roches, minéraux ou ouvrages de paléontologie. Env. oblata.

M. Joseph de Rusunan, fils, à Lez-Plouénan, par Saint-Pol-de-Léon, Finistère, désire échanger plantes, algues, mousses du nord Finistère contre échantillons analogues d'autreș régions.

M. Ph. Rousseau, à la Mazurie, par Aizenay (Vendée) offre: Bons fossiles siluriens et d'autres terrains. Bonnes especes de coquilles marines et terrestres francaises et exotiques, parmi lesquelles quelques raretés, minéraux et roches, plantes marines ét autres cryptogames, phanérogames du littoral du S.-O. et des regions montagneusés de la France, contre échant. analogues, animaux montés, ouvrages d'hist. nat., etc. Env. oblata.

M. Darbois, commis principal des télégraphes, à Oran, offre coquilles du littoral algérien con're coquilles marines principalement exotiques, dénomination bien déterminée, expédition soignée.

M. Launay, instituteur á Saint-Aquilin-de-Pacy (Eure) offre : Parn. Apollo, Col. Phicomone, Lyc. Amandn, Agestor, Mel. Thabe, Selina Ramosa. Tan Atrata, Clrog. Lulcaria, etc., contre autres Lépidoptères de France bien déterminés ou oiseaux montés ou non.

M. A. Boucomont, 20, rue Steffen, à Asnières (Seine) offre : Calosoma sycophanta, inquisitor, Car. victur, monilis et var. Pherosoph. hispanicus, Geotrupes louci, impressus, lavigatus, Unitis pamphyllus, Ancylonicha porosa, Phyllopertha glabra var. noire, Anomala Osmanlis et var. bleue auricollis, rugatipennis, Bolboceras gallicus, Anisoplia tenebralis, Bromicola, Straminea, Dorcadion rufipes, etc.

M. E. André, à Mâcon, offre des cocons vivants de Platisamia cccrepia, désire en échange des cocons de séricigènes ou des papillons de France.

M. Camille Mehier, 6, rue Sainte-Catherine, à Saint-Étienne (Loire) offre des Colćoptères de France contre des plantes de France.

M. Millevoye, 35, rue Malakoff, à Orléans, offre: 12 étaloirs Deyrolles de diverses grosseur's, 60 boîtes en bois liégées pour expérlitions d'insectes, de $0 \mathrm{~m} 20 \times 0 \mathrm{~m} / 15 \times 0 \mathrm{~m}(1 \mathrm{~h} 5$, 300 plaquettes liège d'environ $0^{\mathrm{m}} 16 \times 0^{\mathrm{m}} 04$, le Spectucle de sa Nature, par Pluche, $7 \mathrm{v}$. in-12, reliure veau, avec centaines de planches et gravures, 1780 , demande : coquilles vivantes, grosses et moyennes espèces, silex polis et taillés, plantes vertes et fleurs vivantes.

\section{OUVRAGES OFFERTS A LA BIBLIOTHÈQUE}

DU 9 FÉVRIER $1895^{\circ}$ AU 7 MARS 1896

De la part de : MM. le Dr Aloï ( 1 br.); Cossmann ( 1 br.); prof. Dahl ( 1 br.); prof. i)uboscq (1 br.); prof. Gabelli (1 br.); prof. Giard ( 1 br.); $D^{r}$ Gillot, (3 br.); prof. Griffini (2 b́r.); Dr Maisonneuve (1 br.); Meyran (1 br.); Piette (1 br.); Raspail (1 br.); Schlumberger (3 vol.); prof. Silvestri $(2 \mathrm{br}$.$) .$

Total : 3 volumes, 17 brochures.

Nous adressons tous nos remerciements aux donateurs.

ÉTAT DE LA BIBLIOTHÈQUE AU 7 MARS 1896

$\left.\begin{array}{l}\text { Volumes ................. } 1.777 \\ \text { Brochures............. } 12.550\end{array}\right\}$ sans les recueils scientifiques. 



